\title{
BOUNDS FOR SOLUTIONS OF ORDINARY DIFFERENTIAL EQUATIONS
}

\author{
FRED BRAUER ${ }^{1}$
}

1. An upper bound for the norm of a system of ordinary differential equations can be obtained by comparison with a related first order differential equation, $[4 ; 8]$. This first order equation depends on an upper bound for the norm of the right side of the system. Recently, it has been pointed out $[1 ; 6]$ that this same upper bound also gives a lower bound for the norm of the solution in terms of another first order equation. Most of the known explicit bounds, as well as criteria for global existence and boundedness, can be obtained from such comparison theorems, together with a detailed analysis of the resulting first order equations. The same approach also yields information on the approach of a solution to a limit. As suggested in [1], bounds for approximate local solutions can also be obtained in this way. The bounds given in [1] are sometimes difficult or impossible to calculate explicitly, but it is possible to give slightly weaker bounds, which are more easily calculated.

The main conclusion to be drawn from this paper is that the comparison method provides not only a powerful tool for obtaining bounds for solutions, but also a unified approach to many such problems.

2. Consider the system of ordinary differential equations

$$
x^{\prime}=f(t, x),
$$

where $x$ and $f$ are $n$-dimensional vectors, and $0 \leqq t<\infty$. We assume that $f(t, x)$ is continuous for $0 \leqq t<\infty,|x|<\infty$, but we require no assumptions on $f$ to assure the uniqueness of solutions of (1), as our arguments do not require uniqueness. Suppose that there exists a continuous non-negative function $\omega(t, r)$ on $0 \leqq t<\infty, 0 \leqq r<\infty$, such that

$$
|f(t, x)| \leqq \omega(t,|x|), \quad 0 \leqq t<\infty, \quad|x|<\infty .
$$

It is well-known $[4 ; 8]$ that if $x(t)$ is a solution of $(1)$, and $r(t)$ is the maximum solution of the scalar equation

Presented to the Society, January 24, 1962 under the title On first order differential equations; received by the editors January 16, 1962.

1 Sponsored by the Mathematics Research Center, United States Army, Madison, Wisconsin, under Contract No.: DA-11-022-ORD-2059. 


$$
r^{\prime}=\omega(t, r)
$$

with $r(0)=|x(0)|$, then $x(t)$ can be continued to the right as far as $r(t)$ exists, and

$$
|x(t)| \leqq r(t)
$$

for all such $t$. It can be shown, by the same type of argument, that $|x(t)|$ is also bounded below, as follows.

THEOREM 1. Let $x(t)$ be a solution of (1) and let $\zeta(t)$ be the minimum solution of

$$
\zeta^{\prime}=-\omega(t, \zeta)
$$

with $\zeta(0)=|x(0)|$. Then, for all $t \geqq 0$ such that $x(t)$ exists and $\zeta(t) \geqq 0$, we have

$$
|x(t)| \geqq \zeta(t) .
$$

Since Theorem 1 is essentially contained in Theorem 1 of [1], an explicit proof will not be given here. It may be noted that Theorem 1 can also be derived directly from Lemma 2 of [3], or it can be proved by an argument analogous to the proof of Theorem 8 of this paper. The restriction $\zeta(t) \geqq 0$ is required to ensure that $\omega(t, \zeta(t))$ is defined.

Theorem 1 involves a comparison between solutions of the system (1) and the scalar equation (5). Another comparison theorem arises in the consideration of limiting behavior of solutions.

THEOREM 2. Suppose that (2) is satisfied for a function $\omega(t, r)$ which is monotone nondecreasing in $r$ for each fixed $t$, and suppose that all solutions of (3) exist on $0 \leqq t<\infty$ and tend to limits as $t \rightarrow \infty$. Then all solutions of (1) tend to limits as $t \rightarrow \infty$. If $x(t)$ is a solution of (1) with $\lim _{t \rightarrow \infty} x(t)=X$, if $r(t)$ is the maximum solution of (3) with $r(0)=|x(0)|$, and if $\lim _{t \rightarrow \infty} r(t)=R$, then

$$
|X-x(t)| \leqq R-r(t), \quad 0 \leqq t<\infty .
$$

Proof. The first part of the theorem is contained in $[3$, Theorem $13]$, and we need only prove the inequality (7). If $\bar{t}>t \geqq 0$,

$$
\begin{aligned}
|x(\bar{t})-x(t)| & =\left|\int_{t}^{\bar{t}} f(\tau, x(\tau)) d \tau\right| \leqq \int_{t}^{\bar{t}}|f(\tau, x(\tau))| d \tau \\
& \leqq \int_{t}^{\bar{t}} \omega(\tau,|x(\tau)|) d \tau \leqq \int_{t}^{\bar{\tau}} \omega(\tau, r(\tau)) d \tau \\
& =r(\bar{t})-r(t),
\end{aligned}
$$

using (2) and the monotonicity of $\omega$. The result now follows if we let 
$\bar{t} \rightarrow \infty$ holding $t$ fixed. This proof is only a slight extension of that given in [3, Theorem 4].

3. If the function $\omega(t, r)$ has the form $\lambda(t) \phi(r)$, the condition (2) is replaced by

$$
|f(t, x)| \leqq \lambda(t) \phi(|x|) .
$$

The results of $\$ 2$ lead us to consider the separable first order equations

$$
r^{\prime}=\lambda(t) \phi(r)
$$

and

$$
\zeta^{\prime}=-\lambda(t) \phi(\zeta)
$$

which can be solved explicitly. We define $J(r)=\int_{0}^{r} d u / \phi(u)$. If this integral diverges at zero, the lower limit of integration can be replaced by any fixed $\epsilon>0$, but we will use the lower limit zero for convenience. If $\int_{0}^{\infty} d u / \phi(u)=R \leqq \infty$, the function $J$ maps the half line $[0, \infty)$ onto the interval $[0, R)$, with $J(\infty)=R$. The function $J$ has a positive derivative $1 / \phi(r)$, and is therefore monotone increasing. Thus the inverse function $J^{-1}$ exists and is monotone increasing on $[0, R)$.

If $r(t)$ is a solution of (9) with $r(0)=r_{0}, \int_{0}^{t} \lambda(s) d s=\int_{r_{0}}^{r} d u / \phi(u)$ $=J(r)-J\left(r_{0}\right)$. Thus $J(r)=J\left(r_{0}\right)+\int_{0}^{t} \lambda(s) d s$, and

$$
r(t)=J^{-1}\left[J\left(r_{0}\right)+\int_{0}^{t} \lambda(s) d s\right] .
$$

This solution exists as long as $J\left(r_{0}\right)+\int_{0}^{t} \lambda(s) d s$ is in the domain $[0, R)$ of $J^{-1}$. This requires $J\left(r_{0}\right)+\int_{0}^{t} \lambda(s) d s<R$, or $\int_{0}^{t} \lambda(s) d s<\int_{r_{0}}^{\infty} d u / \phi(r)$. It follows that the solution $r(t)$ of (9) with $r(0)=r_{0}$ exists on the interval $[0, T)$, where $T$ is defined by $\int_{0}^{T} \lambda(s) d s=\int_{r_{0}}^{\infty} d u / \phi(u)$. If $\int_{r_{0}}^{\infty} d u / \phi(u)=\infty$, the solution exists on $0 \leqq t<\infty$. If $\int_{0}^{\infty} \lambda(s) d s<\int_{r_{0}}^{\infty} d u / \phi(u) \leqq \infty$, then $J\left(r_{0}\right)+\int_{0}^{t} \lambda(s) d s<R$, which implies $r(t)<\infty$, and the solution $r(t)$ remains bounded on $0 \leqq t<\infty$. Since $r(t)$ is monotone increasing, this implies that $r(t)$ tends to a limit as $t \rightarrow \infty$.

We can solve (10) by the same approach. We find that the solution $\zeta(t)$ of $(10)$ with $\zeta(0)=r_{0}$ is given by

$$
\zeta(t)=J^{-1}\left[J\left(r_{0}\right)-\int_{0}^{t} \lambda(s) d s\right]
$$

defined so long as $J\left(r_{0}\right)-\int_{0}^{t} \lambda(s) d s \geqq 0$. Thus $\zeta(t)$ exists on the interval $[0, \tau]$, where $\int_{0}^{\gamma} \lambda(s) d s=\int_{0}^{r_{0}} d u / \phi(u)$. If $\int_{0}^{\infty} \lambda(s) d s \leqq \int_{0}^{r_{0}} d u / \phi(u), \zeta(t)$ exists on $0 \leqq t<\infty$, and if $\int_{0}^{\infty} \lambda(s) d s<\int_{0}^{70} d u / \phi(u), \zeta(t)>0$ on $0 \leqq t<\infty$. 
To avoid misinterpretation, recall that we have assumed the convergence of the integral $\int_{0}^{r_{0}} d u / \phi(u)$.

These arguments, combined with the results of $\$ 2$, yield the following known results.

ThEOREM 3 (COOKE [5]). If the condition (8) is satisfied, then all solutions $x(t)$ of (1) with $|x(0)| \leqq r_{0}$ exist on the interval $[0, T)$, where $\int_{0}^{T} \lambda(s) d s=\int_{r_{0}}^{\infty} d u / \phi(u)$. If $\int_{0}^{\infty} \lambda(s) d s \leqq \int_{r_{0}}^{\infty} d u / \phi(u)$, they exist on $0 \leqq t<\infty$, and if $\int_{0}^{\infty} \lambda(s) d s<\int_{r_{0}}^{\infty} d u / \phi(u)$, they are bounded on $[0, \infty)$.

TheOREM 4 (WINTNER [7]). If the condition (8) is satisfied, and $\int_{r_{0}}^{\infty} d u / \phi(u)=\infty$, then all solutions of (1) exist on $0 \leqq t<\infty$. If, in addition, $\int_{0}^{\infty} \lambda(s) d s<\infty$, all solutions of (1) are bounded on $0 \leqq t<\infty$.

THEOREM 5 (BIHARI [2]). If (8) is satisfied, then all solutions $x(t)$ of (1) with $|x(0)| \leqq r_{0}$ obey $|x(t)| \leqq J^{-1}\left[J\left(r_{0}\right)+\int_{0}^{t} \lambda(s) d s\right]$ for all $t$ for which $\int_{0}^{t} \lambda(s) d s \leqq \int_{\tau_{0}}^{\infty} d u / \phi(u)$.

TheOREM 6 (LANGenhop [6]). If (8) is satisfied, then all solutions $x(t)$ of (1) with $|x(0)| \geqq r_{0}$ obey $|x(t)| \geqq J^{-1}\left[J\left(r_{0}\right)-\int_{0}^{t} \lambda(s) d s\right]$ for all $t$ for which $\int_{0}^{t} \lambda(s) d s \leqq \int_{0}^{r_{0}} d u / \phi(u)$.

It follows from Theorem 12 of [3] that any hypotheses which assure the boundedness of all solutions of (9) imply that all solutions of (1) tend to limits as $t \rightarrow \infty$. We can obtain some information about the approach to the limit.

Theorem 7. Suppose that (8) is satisfied with a monotone nondecreasing function $\phi(r)$, and that $\int_{0}^{\infty} \lambda(s) d s<\int_{0}^{\infty} d u / \phi(u)$. Then any solution $x(t)$ of (1) tends to a limit $x(\infty)$ as $t \rightarrow \infty$, and

$$
|x(\infty)-x(t)| \leqq K \int_{t}^{\infty} \lambda(s) d s
$$

where $K=\sup _{t \leq s<\infty} \phi(|x(s)|)=\phi(|x(\infty)|)$.

Proof. Let $r(t)$ be the maximum solution of (9) with $r(0)=|x(0)|$. By Theorem 3, $r(t)$ tends to a limit $r(\infty)$ as $t \rightarrow \infty$, and by Theorem 2, we need only prove

$$
r(\infty)-r(t) \leqq K \int_{t}^{\infty} \lambda(s) d s .
$$

As we have seen, $r(t)$ is given by (11), and thus

$$
r(\infty)=J^{-1}\left[J\left(r_{0}\right)+\int_{0}^{\infty} \lambda(s) d s\right] .
$$


By the mean value theorem, if $0 \leqq \alpha<\beta<R, J^{-1}(\beta)-J^{-1}(\alpha)$ $=(\beta-\alpha)\left(J^{-1}\right)^{\prime}(\xi)$ for some $\xi, \alpha<\xi<\beta$. By the inverse function theorem, $\left(J^{-1}\right)^{\prime}(\xi)=1 / J^{\prime}\left(J^{-1}(\xi)\right)$. It follows from the definition of $J$ that $J^{-1}(\xi)=\phi(\sigma)$, where $\sigma$ is chosen so that $\xi=\phi(\sigma)$. Subtraction of (13) from (11) gives

$$
r(\infty)-r(t)=\phi(\sigma)\left[\int_{0}^{\infty} \lambda(s) d s-\int_{0}^{t} \lambda(s) d s\right]=\phi(\sigma) \int_{t}^{\infty} \lambda(s) d s .
$$

Here, $\sigma$ is chosen so that $J(\sigma)=\xi$, where $\int_{0}^{t} \lambda(s) d s<\xi<\int_{0}^{\infty} \lambda(s) d s$. We can choose $T, t<T<\infty$, so that $\xi=\int_{0}^{T} \lambda(s) d s$, and then $J(\sigma)=\int_{r_{0}}^{\sigma} d u / \phi(u)$ $=\int_{0}^{T} \lambda(s) d s$. This implies $\sigma=r(T), \phi(\sigma) \leqq K$, and completes the proof of (12).

4. These arguments can also be used to study bounds for approximate solutions. A continuous function $x(t)$ is said to be an $\epsilon$-approximate solution of (1) on an interval if it is differentiable on the interval except for a finite set of points, and $\left|x^{\prime}(t)-f(t, x(t))\right|<\epsilon$ on the interval except for this finite set of points. It is understood that this exceptional set is empty if $\epsilon=0$. We assume in this section that $f$ obeys an inequality of the form

$$
|f(t, x)-f(t, y)| \leqq \omega(t,|x-y|),
$$

in some region $0 \leqq t<a,|x|<A,|y|<A,|x-y|<A$, where $\omega(t, r)$ is continuous and non-negative on $0 \leqq t<a, 0 \leqq r<A$. The following result is a special case of a theorem of Antosiewicz [1]. Since the proof of this special case is simpler than the proof of the more general result, we include it here.

THEOREM 8. Let $x_{i}(t)$ be an $\epsilon_{i}$-approximate solution of (1) on $0 \leqq t<a$ $(i=1,2)$, and let $\epsilon=\epsilon_{1}+\epsilon_{2}$. Let $r_{\epsilon}(t)$ be the maximum solution of

$$
r^{\prime}=\omega(t, r)+\epsilon
$$

on $0 \leqq t<a$ with $r_{\epsilon}(0)=\left|x_{1}(0)-x_{2}(0)\right|$, and let $\zeta_{\text {( }}(t)$ be the minimum solution of

$$
\zeta^{\prime}=-\omega(t, \zeta)-\epsilon
$$

on $0 \leqq t<a$ with $\zeta_{\mathrm{e}}(0)=\left|x_{1}(0)-x_{2}(0)\right|$. If (14) is satisfied, then

$$
\zeta_{\epsilon}(t) \leqq\left|x_{1}(t)-x_{2}(t)\right| \leqq r_{\epsilon}(t), \quad 0 \leqq t<a .
$$

Proof. Let $m(t)=\left|x_{1}(t)-x_{2}(t)\right|$. Then $\left|m^{\prime}(t)\right| \leqq\left|x_{1}^{\prime}(t)-x_{2}^{\prime}(t)\right| \leqq\left|f\left(t, x_{1}(t)\right)-f\left(t, x_{2}(t)\right)\right|+\epsilon_{1}+\epsilon_{2}$, and this implies, using (14) and $\epsilon=\epsilon_{1}+\epsilon_{2}$, that 


$$
-\omega(t, m(t))-\epsilon \leqq m^{\prime}(t) \leqq \omega(t, m(t))+\epsilon .
$$

To prove $m(t) \leqq r_{\epsilon}(t)$, it suffices to prove $m(t) \leqq r_{\alpha}(t)$ for all $\alpha>\epsilon$, since $\lim _{\alpha \rightarrow e+} r_{\alpha}(t)=r_{\epsilon}(t)$ for $t \geqq 0$. Suppose this inequalty is false for some $\alpha>\epsilon$, and let $\sigma$ be the greatest lower bound of values of $t$ for which $m(t)>r_{\alpha}(t)$. Since $m(t)$ and $r_{\alpha}(t)$ are continuous, $m(\sigma)=r_{\alpha}(\sigma)$ and $m^{\prime}(\sigma) \geqq r_{\alpha}^{\prime}(\sigma)$. But (18) gives $\omega(\sigma, m(\sigma))+\epsilon \geqq m^{\prime}(\sigma) \geqq r_{\alpha}^{\prime}(\sigma)$ $=\omega\left(\sigma, r_{\alpha}(\sigma)\right)+\alpha$. Since $\alpha>\epsilon$, this is a contradiction, which proves $m(t) \leqq r_{\epsilon}(t)$.

The other part of (17) is proved in a similar manner. Again, it suffices to prove $m(t) \geqq \zeta_{\alpha}(t)$ for all $\alpha>\epsilon$, since $\lim _{\alpha \rightarrow \epsilon+} \zeta_{\alpha}(t)=\zeta_{\epsilon}(t)$ for $t \geqq 0$. If the inequality is false for some $\alpha>\epsilon$, let $\sigma$ be the greatest lower bound of values of $t$ for which $m(t)<\zeta_{\alpha}(t)$. As before, $m(\sigma)$ $=\zeta_{\alpha}(\sigma)$ and $m^{\prime}(\sigma) \leqq \zeta_{\alpha}^{\prime}(\sigma)$, but

$$
-\omega(\sigma, m(\sigma))-\epsilon \leqq m^{\prime}(\sigma) \leqq \zeta_{\alpha}^{\prime}(\sigma)=-\omega\left(\sigma, \zeta_{\alpha}(\sigma)\right)-\alpha,
$$

which contradicts $\alpha>\epsilon$. This proves $m(t) \geqq \zeta_{\epsilon}(t)$ and completes the proof of (17).

Theorem 8 compares approximate solutions of a system to exact solutions of an approximate scalar equation. It is often more convenient to compare the approximate solutions to exact solutions of an exact scalar equation with approximate initial conditions. Although the bounds obtained in this way are less precise, they are considerably easier to calculate.

Let $r\left(t, r_{0}\right)$ be the maximum solution of (3) with $r\left(0, r_{0}\right)=r_{0}$, and let $r_{e}(t)$ be the maximum solution of (15) with $r_{e}(0)=r_{0}$.

TheOREM 9. If $\omega(t, r)$ is monotone nondecreasing in $r$ for each fixed $t$, then for any fixed $\epsilon>0, r_{\epsilon}(t) \leqq r\left(t, r_{0}+\epsilon t\right)$ for $t \geqq 0$.

Proof. From (3) and (15) we obtain

$$
\begin{gathered}
r_{\epsilon}(t)=r_{0}+\int_{0}^{t} \omega\left(s, r_{\epsilon}(s)\right) d s+\epsilon t \\
r\left(t, r_{0}+\epsilon t\right)=r_{0}+\epsilon t+\int_{0}^{t} \omega\left(s, r\left(s, r_{0}+\epsilon t\right)\right) d s .
\end{gathered}
$$

Since $\omega$ is assumed monotone, the theorem will follow from

$$
r_{\epsilon}(s) \leqq r\left(s, r_{0}+\epsilon t\right), \quad 0 \leqq s \leqq t .
$$

If (19) is false, there exists $\sigma, 0 \leqq \sigma<t$, such that $\sigma$ is the greatest lower bound of values of $s$ for which $r_{\epsilon}(s)>r\left(s, r_{0}+\epsilon t\right)$. Since both functions are continuous, $r_{\epsilon}(\sigma)=r\left(\sigma, r_{0}+\epsilon t\right)$. But $r_{\epsilon}(\sigma)=r_{0}$ $+\int_{0}^{\sigma} \omega\left(u, r_{\epsilon}(u)\right) d u+\epsilon \sigma$, and $r\left(\sigma, r_{0}+\epsilon t\right)=r_{0}+\epsilon t+\int_{0}^{\sigma} \omega\left(u, r\left(u, r_{0}+\epsilon t\right)\right) d u$. 
Since $\omega$ is monotone, $r_{\epsilon}(u) \leqq r\left(u, r_{0}+\epsilon t\right)$ for $0 \leqq u \leqq \sigma$, and $\sigma<t$, (19) follows.

Theorem 9 has an analogue for lower bounds, proved in the same way. Let $\zeta\left(t, r_{0}\right)$ be the minimum solution of (5) with $\zeta\left(0, r_{0}\right)=r_{0}$, and let $\zeta_{\epsilon}(t)$ be the minimum solution of $(16)$ with $\zeta_{\epsilon}(0)=r_{0}$.

THEOREM 10. If $\omega(t, r)$ is monotone nondecreasing in $r$ for each fixed $t$, then for any fixed $\epsilon>0$

$$
\zeta_{\mathrm{e}}(t) \geqq \zeta\left(t, r_{0}-\epsilon t\right), \quad t \geqq 0 .
$$

If $f(t, x)$ satisfies a Lipschitz condition, we can take $\omega(t, r)=K r$. In this case, Theorem 8 and the solution of $r^{\prime}=K r+\epsilon$ give upper and lower bounds for $\left|x_{1}(t)-x_{2}(t)\right|$, where $x_{i}(t)$ is an $\epsilon_{i}$-approximate solution of (1) for $i=1,2$, and $\epsilon=\epsilon_{1}+\epsilon_{2}$.

$$
\begin{aligned}
\left|x_{1}(0)-x_{2}(0)\right| e^{-K t}-\frac{\epsilon}{K}\left(1-e^{-K t}\right) & \\
& \leqq\left|x_{1}(t)-x_{2}(t)\right| \leqq\left|x_{1}(0)-x_{2}(0)\right| e^{K t}+\frac{\epsilon}{K}\left(e^{K t}-1\right) .
\end{aligned}
$$

The upper bound is classical, while the lower bound is due to Antosiewicz [1]. If we apply Theorem 9, which requires solving $r^{\prime}=K r$, we obtain

$$
\begin{aligned}
\left|x_{1}(0)-x_{2}(0)\right| e^{-K t}-\epsilon t e^{-K t} & \leqq\left|x_{1}(t)-x_{2}(t)\right| \\
& \leqq\left|x_{1}(0)-x_{2}(0)\right| e^{K t}+\epsilon t e^{K t}
\end{aligned}
$$

These bounds are weaker than the earlier ones by a term which is $0\left(\epsilon t^{2}\right)$ for small $t$.

If $f$ satisfies an inequality

$$
|f(t, x)-f(t, y)| \leqq \lambda(t) \phi(|x-y|),
$$

application of Theorem 8 would require the solution of $r^{\prime}=\lambda(t) \phi(r)$ $+\epsilon$, which cannot be found explicitly in general. The use of Theorem 9 , however, requires only the solution of $r^{\prime}=\lambda(t) \phi(r)$, which has been obtained in §3. This yields the following bounds. The upper bound has been obtained previously by Bihari [2].

Theorem 11. If $x_{i}(t)$ are $\epsilon_{i}$-approximate solutions of (1) for $i=1,2$, if $\epsilon=\epsilon_{1}+\epsilon_{2}$, if $\left|x_{1}(0)-x_{2}(0)\right| \leqq r_{0}$, and if $f$ satisfies (20), then

$$
\begin{aligned}
J^{-1}\left[J\left(r_{0}-\epsilon t\right)-\int_{0}^{t} \lambda(s) d s\right] & \leqq\left|x_{1}(t)-x_{2}(t)\right| \\
& \leqq J^{-1}\left[J\left(r_{0}+\epsilon t\right)+\int_{0}^{t} \lambda(s) d s\right],
\end{aligned}
$$


where $J(r)=\int_{0}^{r} d u / \phi(u)$, and $J^{-1}$ is the inverse function, as in $\S 3$.

We can apply Theorem 11 when $x_{1}(t), x_{2}(t)$ are exact solutions of (1) with $x_{1}(0)=x_{2}(0)$. This means taking $\epsilon=0, r_{0}=0$. We obtain

$$
J^{-1}\left[J(0)-\int_{0}^{t} \lambda(s) d s\right] \leqq\left|x_{1}(t)-x_{2}(t)\right| \leqq J^{-1}\left[J(0)+\int_{0}^{t} \lambda(s) d s\right] .
$$

If $\int_{0} d u / \phi(u)$ converges, $J(0)$ is defined, and $J(0)=0$. The lower bound becomes vacuous, since $J(0)-\int_{0}^{t} \lambda(s) d s \leqq 0$ for $t \geqq 0$, but we still have an upper bound

$$
\left|x_{1}(t)-x_{2}(t)\right| \leqq J^{-1}\left[\int_{0}^{t} \lambda(s) d s\right]
$$

If $\int_{0} d u / \phi(u)$ diverges, $J(0)$ is not defined, and our arguments cannot be used without considerable modification. In this case, however, the Osgood theorem shows that $x_{1}(t)-x_{2}(t)=0$ on $0 \leqq t<a$. The inequality (21) gives a bound in the nonunique case.

\section{BiBLIOGRAPHY}

1. H. A. Antosiewicz, An inequality for approximate solutions of ordinary differential equations, Math. Z. 78 (1962), 44-52.

2. I. Bihari, $A$ generalization of a lemma of Bellman and its application to uniqueness problems of differential equations, Acta Math. Sci. Hungar. 7 (1956), 81-94.

3. F. Brauer, Global behavior of solutions of ordinary differential equations, J. Math. Anal. Appl. 2 (1961), 145-158.

4. R. Conti, Sulla prolungabilita delle soluzioni di un sistema di equazioni differenziali ordinarie, Boll. Un. Math. Ital. 11 (1956), 510-514.

5. K. L. Cooke, A non-local existence theorem for systems of ordinary differential equations, Rend. Circ. Mat. Palermo 4 (1955), 301-308.

6. C. E. Langenhop, Bounds on the norm of $a$ solution of a general differential equation, Proc. Amer. Math. Soc. 11 (1960), 795-799.

7. A. Wintner, An abelian lemma concerning asymptotic equilibria, Amer. J. Math. 78 (1946), 451-454.

8. - Ordinary differential equations and Laplace transforms (appendix), Amer. J. Math. 79 (1957), 265-294.

UNIVERSITY OF WISCONSIN 